\title{
On a sum form functional equation emerging from statistics and its applications
}

\author{
Dhiraj Kumar Singha , Shveta Grover ${ }^{b}$ \\ a Department of Mathematics, Zakir Husain Delhi College (University of Delhi), Jawaharlal Nehru Marg, Delhi 110002, India. \\ ${ }^{b}$ Department of Mathematics, University of Delhi, Delhi 110007, India.
}

\begin{abstract}
In this paper, we obtain the general solutions of a sum form functional equation arising from the expected value of a discrete random variable. The significance of its general solutions in reference to entropies emerging from information theory and diversity index has been discussed.
\end{abstract}

Keywords: Additive mapping; bounded mapping; linear mapping; logarithmic mapping; the entropies of type $(\alpha, \beta)$; diversity index; expectation of a discrete random variable.

$2010 M S C:$ 39B52, 39B82.

\section{Introduction}

Over the years, research in the field of functional equations with reference to information theory has evolved into studying those functional equations which are useful in characterizing entropies. The study of these entropies is interesting as well as demanding since they have been used in a wide variety of studies in Economics (Gini [4]); Ecology (Pielou [25]); Biology (Lewontin [11]) and many more. Thus this integrative approach of functional equations inspired us to explore those functional equations which not only characterizes entropies but are related to new branches too. In this paper, we have identified a functional equation arising from statistics and obtained its general solutions. Further, we have tried to establish a connect of this functional equation with information theory and diversity index.

Throughout the paper, let $\mathbb{R}$ denote the set of real numbers; $I$ denote the closed interval $[0,1]$. For $n=1,2, \ldots$; let

$$
\Gamma_{n}=\left\{\left(p_{1}, \ldots, p_{n}\right) ; p_{i} \geq 0, i=1, \ldots, n ; \sum_{i=1}^{n} p_{i}=1\right\}
$$

Email addresses: dksingh@zh.du.ac.in, dhiraj426@rediffmail.com (Dhiraj Kumar Singh), srkgrover9@gmail.com (Shveta Grover) 
denote the set of all finite $n$-component discrete probability distributions.

For a given probability distribution $\left(p_{1}, \ldots, p_{n}\right) \in \Gamma_{n}$, consider a real valued discrete random variable $X_{n}$ taking $n$ distinct values $x_{1}, \ldots, x_{n}$ with probabilities $p_{1}, \ldots, p_{n}$ where

$$
x_{i}= \begin{cases}-p_{i}^{\beta-1} \log _{2} p_{i} & \text { if } 0<p_{i} \leq 1 \\ 0 & \text { if } p_{i}=0\end{cases}
$$

such that $\beta$ is a fixed positive real power with $0^{\beta}:=0,1^{\beta}:=1$ and $0^{\beta-1} \log _{2} 0:=0$. If we fix $\beta=1$, then the above random variable $X_{n}$ reduces to the random variable $Z_{n}$ taking different real values $z_{1}, \ldots, z_{n}$ with respective probabilities $p_{1}, \ldots, p_{n}$ where

$$
z_{i}= \begin{cases}-\log _{2} p_{i} & \text { if } 0<p_{i} \leq 1 \\ 0 & \text { if } p_{i}=0\end{cases}
$$

This random variable $Z_{n}$ has been discussed in detail by Nath and Singh [21. This random variable $Z_{n}$ is closely related to Shannon entropies [27]. In this paper we fix $\beta \neq 1$ and discuss the random variable $X_{n}$. Then expected value [16] $E\left(X_{n}\right)$ of the random variable $X_{n}$ is

$$
E\left[X_{n}\right]=-\sum_{i=1}^{n} p_{i}^{\beta} \log _{2} p_{i} .
$$

Define the mapping $\psi_{(\beta, r)}: I \rightarrow \mathbb{R}, r=1,2, \ldots$ as

$$
\psi_{(\beta, r)}(p)=-p^{\beta}\left(\log _{2} p\right)^{r}
$$

for all $p \in I$ and $0^{\beta}\left(\log _{2} 0\right)^{r}:=0$. Furthermore for $r=1,2, \ldots$, it follows that

$$
\psi_{(\beta, r)}(0)=0 \quad \text { and } \quad \psi_{(\beta, r)}(1)=0 .
$$

Clearly with the help of 1.1 and 1.2 , we get

$$
E\left[X_{n}\right]=-\sum_{i=1}^{n} \psi_{(\beta, 1)}\left(p_{i}\right)
$$

Thus expected value of random variable $X_{n}$ has a sum form representation given by $\psi_{(\beta, 1)}$. As a result the mapping $\psi_{(\beta, 1)}$ is called its generating function [16].

Now substituting $r=1$ in $(1.2)$ and using $0^{\beta} \log _{2} 0:=0$, it can be verified easily that mapping $\psi_{(\beta, 1)}$ satisfy the functional equation

$$
\psi_{(\beta, 1)}(p q)=p^{\beta} \psi_{(\beta, 1)}(q)+q^{\beta} \psi_{(\beta, 1)}(p)
$$

for all $p \in I, q \in I$ with $\psi_{(\beta, 1)}(0)=0$ and $\psi_{(\beta, 1)}(1)=0$. As a result for all $\left(p_{1}, \ldots, p_{n}\right) \in \Gamma_{n},\left(q_{1}, \ldots, q_{m}\right) \in$ $\Gamma_{m}$, the functional equation

$$
\sum_{i=1}^{n} \sum_{j=1}^{m} \psi_{(\beta, 1)}\left(p_{i} q_{j}\right)=\sum_{i=1}^{n} p_{i}^{\beta} \sum_{j=1}^{m} \psi_{(\beta, 1)}\left(q_{j}\right)+\sum_{j=1}^{m} q_{j}^{\beta} \sum_{i=1}^{n} \psi_{(\beta, 1)}\left(p_{i}\right)
$$

holds for an arbitrary pair $(n, m)$ of positive integers where $\beta \neq 1,0^{\beta}:=0,1^{\beta}:=1 ; \psi_{(\beta, 1)}(0)=0$ and $\psi_{(\beta, 1)}(1)=0$.

With the aid of (1.4), it can be concluded that $(1.5)$ is emerging from statistics. So, it seems worthy to pay attention to this functional equation. Interestingly, Behara and Nath 2] were first who came across functional equation (1.5) as they considered its generalized form which is

$$
\sum_{i=1}^{n} \sum_{j=1}^{m} f\left(p_{i} q_{j}\right)=\sum_{i=1}^{n} p_{i}^{\alpha} \sum_{j=1}^{m} f\left(q_{j}\right)+\sum_{j=1}^{m} q_{j}^{\beta} \sum_{i=1}^{n} f\left(p_{i}\right)
$$


where $f: I \rightarrow \mathbb{R}$ is an unknown mapping; $\left(p_{1}, \ldots, p_{n}\right) \in \Gamma_{n},\left(q_{1}, \ldots, q_{m}\right) \in \Gamma_{m} ; n \geq 3, m \geq 3$ be fixed integers; $\alpha \neq 1$ and $\beta \neq 1$ are fixed positive real powers satisfying

$$
0^{\alpha}:=0,0^{\beta}:=0,1^{\alpha}:=1,1^{\beta}:=1 .
$$

The functional equation (1.6), is useful in characterizing the nonadditive entropies of type $(\alpha, \beta)$ given by Behara and Nath. For a probability distribution $\left(p_{1}, \ldots, p_{n}\right) \in \Gamma_{n}$, it is defined as follows:

$$
H_{n}^{(\alpha, \beta)}\left(p_{1}, \ldots, p_{n}\right)= \begin{cases}\left(2^{1-\alpha}-2^{1-\beta}\right)^{-1}\left(\sum_{i=1}^{n} p_{i}^{\alpha}-\sum_{i=1}^{n} p_{i}^{\beta}\right) & \text { if } \alpha \neq \beta \\ -2^{\beta-1} \sum_{i=1}^{n} p_{i}^{\beta} \log _{2} p_{i} & \text { if } \alpha=\beta\end{cases}
$$

where $H_{n}^{(\alpha, \beta)}: \Gamma_{n} \rightarrow \mathbb{R}, n=1,2, \ldots ; 0^{\beta} \log _{2} 0:=0$ and $\alpha, \beta$ are fixed positive real powers satisfying (1.7).

Behara and Nath were first to study $(1.6)$ and obtained its continuous solutions by presuming $f: I \rightarrow \mathbb{R}$ to be continuous with $0<\alpha, \beta \in \mathbb{R}$ and $n, m=1,2, \ldots$ Later on, Kannappan [8] obtained its integrable solutions by presuming $f: I \rightarrow \mathbb{R}$ to be integrable and $n=3, m=2$. In the following year, Kannappan [9] obtained its measurable solutions by presuming $f: I \rightarrow \mathbb{R}$ to be Lebesgue measurable and $n=m=3$. Eventually, Losonczi and Maksa [13] without imposing any regularity conditions on the mapping $f: I \rightarrow \mathbb{R}$ found the general solutions of 1.6 for $n \geq 3, m \geq 2$ being fixed integers with $\alpha \neq 1, \beta \neq 1$.

It appears that over the last few years functional equation $(1.6)$ was addressed several times and studied extensively. Kocsis [10] was first to consider a generalization of (1.6) by replacing its power mappings with multiplicative mappings $M_{1}: I \rightarrow \mathbb{R}, M_{2}: I \rightarrow \mathbb{R}$ and studying the functional equation

$$
\sum_{i=1}^{n} \sum_{j=1}^{m} f\left(p_{i} q_{j}\right)=\sum_{i=1}^{n} M_{1}\left(p_{i}\right) \sum_{j=1}^{m} f\left(q_{j}\right)+\sum_{j=1}^{m} M_{2}\left(q_{j}\right) \sum_{i=1}^{n} f\left(p_{i}\right)
$$

where $f: I \rightarrow \mathbb{R}$ is an unknown mapping; $\left(p_{1}, \ldots, p_{n}\right) \in \Gamma_{n},\left(q_{1}, \ldots, q_{m}\right) \in \Gamma_{m}$. He obtained its general solutions and discussed its stability for $n \geq 3, m \geq 3$ being fixed integers. Thereafter, Nath and Singh [20], [23]; Singh and Dass [28] discussed a Pexiderized form of (1.6) where they obtained the general solutions of the functional equation for $n \geq 3, m \geq 3$ being fixed integers. Singh and Grover [29] discussed the stability problem for a Pexiderized form of 1.6 for $n \geq 3, m \geq 3$ being fixed integers. Apart from these, few significant contributions that have appeared in the field of sum form functional equations which are characterizing various entropies are as follows: Nath and Singh [17] obtained the general solutions of a multiplicative type functional equation which is useful in the characterization of the nonadditive measure of entropy proposed by Havrda and Charvát [5]. Building upon their work, Nath and Singh [18] considered an extended form of the multiplicative type functional equation (discussed in [17]) and demonstrated the significance of its solutions with Shannon [27]; Havrda and Charvát [5] entropies. A similar result was established by Nath and Singh in [19] and [22] by addressing functional equations containing only two unknown mappings.

It needs to be remarked that most of these results were from information theoretic point of view and sum form functional equations emerging from statistics are relatively less explored. In this paper, we have tried establishing its relation with the expected value of a discrete random variable (following from (1.4) thus, adding a new dimension to functional equation (1.6). Moreover, one of its Pexiderized form which seems to have been missed by the researchers is as follows:

$$
\sum_{i=1}^{n} \sum_{j=1}^{m} f\left(p_{i} q_{j}\right)=\sum_{i=1}^{n} p_{i}^{\alpha} \sum_{j=1}^{m} g\left(q_{j}\right)+\sum_{j=1}^{m} q_{j}^{\beta} \sum_{i=1}^{n} h\left(p_{i}\right)
$$

where $f: I \rightarrow \mathbb{R}, g: I \rightarrow \mathbb{R}, h: I \rightarrow \mathbb{R}$ are unknown mappings; $\left(p_{1}, \ldots, p_{n}\right) \in \Gamma_{n},\left(q_{1}, \ldots, q_{m}\right) \in \Gamma_{m} ; n \geq 3$, $m \geq 3$ be fixed integers; $\alpha \neq 1$ and $\beta \neq 1$ are fixed positive real powers satisfying (1.7). 
Clearly, it can be concluded that functional equation $(\mathrm{A})$ is emerging from statistics and also useful in providing characterization of the entropies of type $(\alpha, \beta)$ given by $(1.8)$. Therefore, it seems desirable to study functional equation (A).

Also, while obtaining solutions of functional equation $(\mathrm{A})$ we came across a new open problem (unfolding from statistics), which is

$$
\begin{gathered}
\sum_{i=1}^{n} \sum_{j=1}^{m} G\left(p_{i} q_{j}\right)=\sum_{i=1}^{n} p_{i}^{\alpha} \sum_{j=1}^{m} G\left(q_{j}\right)+\sum_{j=1}^{m} q_{j}^{\beta} \sum_{i=1}^{n} G\left(p_{i}\right) \\
+(m-n) G(0) \sum_{j=1}^{m} q_{j}^{\beta}+m(n-1) G(0)
\end{gathered}
$$

where $G: I \rightarrow \mathbb{R}$ is an unknown mapping; $\left(p_{1}, \ldots, p_{n}\right) \in \Gamma_{n},\left(q_{1}, \ldots, q_{m}\right) \in \Gamma_{m} ; n \geq 3, m \geq 3$ be fixed integers; $\alpha \neq 1$ and $\beta \neq 1$ are fixed positive real powers satisfying the conventions mentioned in (1.7). The functional equation $(\sqrt{B})$ was first observed by Nath and Singh (with $G$ in place of $f$, see p. 33, [24]). Although they mentioned it to be an enlargement of (1.6) but still left it unsolved. Consequently functional equation (B) becomes significant and need to be discussed.

Summarizing so far, it can be concluded that we have arrived at two open problems i.e. functional equations $(\mathrm{A})$ and $(\mathrm{B})$ both arising from statistics and characterizing entropies as well. Hence both functional equations are connecting two different branches of research which are information theory and mathematical statistics. This provides us sufficient reasons to study functional equations $(A)$ and $(B)$. Thus, our goal in this paper is to obtain the general solutions of $(A)$ and $(B)$ without imposing any regularity conditions on the mappings appearing in them.

This paper is structured as follows:

In section 1, we have established the relation between functional equations and statistics, from which we have arrived at functional equations $(\mathrm{A})$ and $(\mathrm{B})$. In section 2 , we state few known results and definitions which will be used in the subsequent sections. In section 3, we obtain general solutions of the functional equations $(\mathrm{A})$ and $(\mathrm{B})$ for $n \geq 3, m \geq 3$ being fixed integers. In section 4 , we discuss the significance of the general solutions obtained in section 3 with reference to information theory and diversity index.

\section{Auxiliary results}

We begin this section by stating some definitions followed by results, which we will be using in the subsequent sections.

A mapping $a: I \rightarrow \mathbb{R}$ is said to be additive on $I$ if it satisfies $a(x+y)=a(x)+a(y)$ for all $x \in I$, $y \in I$. Similarly, a mapping $A: \mathbb{R} \rightarrow \mathbb{R}$ is said to be additive on $\mathbb{R}$ if it satisfies $A(x+y)=A(x)+A(y)$ for all $x \in \mathbb{R}, y \in \mathbb{R}$. Further, Daróczy and Losonczi [3] established an interesting connect between these two additive mappings and proved that there exists a unique additive extension of the additive mapping $a: I \rightarrow R$ to the set of real numbers.

A mapping $\ell: I \rightarrow \mathbb{R}$ is said to be logarithmic on $I$ if it satisfies $\ell(0)=0$ and $\ell(x y)=\ell(x)+\ell(y)$ for all $x \in] 0,1], y \in] 0,1]$.

A mapping $m: I \rightarrow \mathbb{R}$ is said to be multiplicative on $I$ if it satisfies $m(0)=0, m(1)=1$ and $m(x y)=$ $m(x) m(y)$ for all $x \in] 0,1[, y \in] 0,1[$.

Lemma 1 ([14]). Let $n \geq 3$ be a fixed integer and $\phi: I \rightarrow \mathbb{R}$ be a mapping which satisfy the functional equation $\sum_{i=1}^{n} \phi\left(p_{i}\right)=\bar{c}$ for all $\left(p_{1}, \ldots, p_{n}\right) \in \Gamma_{n} ; \bar{c}$ a given real constant. Then there exists an additive mapping $A_{1}: \mathbb{R} \rightarrow \mathbb{R}$ such that $\phi(p)=A_{1}(p)-\frac{1}{n} A_{1}(1)+\frac{\bar{c}}{n}$ for all $p \in I$.

Lemma 2 ([10]). If an additive mapping $A_{2}: \mathbb{R} \rightarrow \mathbb{R}$ and a multiplicative mapping $M: I \rightarrow \mathbb{R}$ satisfy $A_{2}(p)=M(p)+c_{0} ; c_{0}$ a given real constant, then there exists some $c^{*} \in \mathbb{R}$ such that $A_{2}(p)=c^{*} p$ for all $p \in \mathbb{R} ; c_{0}=0$ and $M(p)=0$ or $M(p)=p$ for all $p \in I$. 
Lemma 3 ([10]). If an additive mapping $A_{3}: \mathbb{R} \rightarrow \mathbb{R}$ and multiplicative mappings $M_{1}: I \rightarrow \mathbb{R}, M_{2}: I \rightarrow \mathbb{R}$ with $M_{1} \neq M_{2}$ satisfy $A_{3}(p)+d=M_{1}(p)-M_{2}(p) ; d$ being a real constant, then $M_{1}$ and $M_{2}$ are identity or zero mappings on $I$.

\section{The general solution of functional equations $A$ and $(B)$}

The main result of this section is as follows:

Theorem 3.1. Let $n \geq 3, m \geq 3$ be fixed integers and $f: I \rightarrow \mathbb{R}, g: I \rightarrow \mathbb{R}, h: I \rightarrow \mathbb{R}$ be mappings which satisfy the functional equation (A) for all $\left(p_{1}, \ldots, p_{n}\right) \in \Gamma_{n},\left(q_{1}, \ldots, q_{m}\right) \in \Gamma_{m} ; \alpha \neq 1$ and $\beta \neq 1$ be fixed positive real powers satisfying (1.7). Then for all $p \in I$, any general solution $(f, g, h)$ of A is of the form (for $\alpha=\beta$ )

$$
\begin{aligned}
& \text { (i) } \left.\quad f(p)=p^{\beta} \ell(p)+[g(1)+(m-1) g(0)+h(1)+(n-1) h(0)] p^{\beta}\right) \\
& +\bar{E}_{0}(p)+f(0), \bar{E}_{0}(1)=-n m f(0), \\
& \text { (ii) } g(p)=p^{\beta} \ell(p)+[g(1)+(m-1) g(0)] p^{\beta}+E_{0}(p)+g(0) \text {, } \\
& E_{0}(1)=-m g(0), \\
& \text { (iii) } h(p)=p^{\beta} \ell(p)+[h(1)+(n-1) h(0)] p^{\beta}+E_{0}^{*}(p)+h(0) \text {, } \\
& E_{0}^{*}(1)=-n h(0)
\end{aligned}
$$

and $($ for $\alpha \neq \beta)$

$$
\left.\begin{array}{rl}
\text { (i) } \quad f(p)= & d\left(p^{\alpha}-p^{\beta}\right)+[g(1)+(m-1) g(0)+h(1)+(n-1) h(0)] p^{\beta} \\
& +\bar{E}_{1}(p)+f(0), \bar{E}_{1}(1)=-n m f(0), \\
\text { (ii) } g(p)= & d\left(p^{\alpha}-p^{\beta}\right)+[g(1)+(m-1) g(0)] p^{\beta}+E_{1}(p)+g(0), \\
& E_{1}(1)=-m g(0), \\
\text { (iii) } h(p)= & \{d-[g(1)+(m-1) g(0)]\}\left(p^{\alpha}-p^{\beta}\right) \\
& +[h(1)+(n-1) h(0)] p^{\beta}+E_{1}^{*}(p)+h(0), E_{1}^{*}(1)=-n h(0)
\end{array}\right\}
$$

where $\bar{E}_{0}: \mathbb{R} \rightarrow \mathbb{R}, E_{0}: \mathbb{R} \rightarrow \mathbb{R}, E_{0}^{*}: \mathbb{R} \rightarrow \mathbb{R}, \bar{E}_{1}: \mathbb{R} \rightarrow \mathbb{R}, E_{1}: \mathbb{R} \rightarrow \mathbb{R}$ and $E_{1}^{*}: \mathbb{R} \rightarrow \mathbb{R}$ are additive mappings $\ell: I \rightarrow \mathbb{R}$ is a logarithmic mapping and $d$ is an arbitrary real constant.

Before giving the proof of this theorem, we need to obtain the general solutions of the functional equation (B). So, we provide it in the following lemma:

Lemma 4. Let $n \geq 3, m \geq 3$ be fixed integers and $G: I \rightarrow \mathbb{R}$ be a mapping which satisfy the functional equation (B) for all $\left(p_{1}, \ldots, p_{n}\right) \in \Gamma_{n},\left(q_{1}, \ldots, q_{m}\right) \in \Gamma_{m} ; \alpha \neq 1$ and $\beta \neq 1$ be fixed positive real powers satisfying (1.7). Then for all $p \in I$, any general solution of $(\mathrm{B})$ is of the form (for $\alpha=\beta$ )

$$
G(p)=p^{\beta} \ell(p)+E_{0}(p)+G(0), E_{0}(1)=-m G(0)
$$

and $($ for $\alpha \neq \beta)$

$$
G(p)=d\left(p^{\alpha}-p^{\beta}\right)+E_{1}(p)+G(0), E_{1}(1)=-m G(0)
$$

where $E_{0}: \mathbb{R} \rightarrow \mathbb{R}$ and $E_{1}: \mathbb{R} \rightarrow \mathbb{R}$ are additive mappings; $\ell: I \rightarrow \mathbb{R}$ is a logarithmic mapping; $d$ is an arbitrary real constant.

Proof. By applying Lemma 1 on the functional equation $(\mathrm{B})$, there exists a mapping $E: \Gamma_{n} \times \mathbb{R} \rightarrow \mathbb{R}$, additive in the second variable such that

$$
\begin{aligned}
\sum_{i=1}^{n} G\left(p_{i} q\right) & -[G(q)-G(0)] \sum_{i=1}^{n} p_{i}^{\alpha}-q^{\beta}\left[\sum_{i=1}^{n} G\left(p_{i}\right)+(m-n) G(0)\right]-n G(0) \\
= & E\left(p_{1}, \ldots, p_{n} ; q\right)
\end{aligned}
$$


with

$$
E\left(p_{1}, \ldots, p_{n} ; 1\right)=-m G(0)\left[1-\sum_{i=1}^{n} p_{i}^{\alpha}\right] .
$$

Let $x \in I$ and $\left(r_{1}, \ldots, r_{n}\right) \in \Gamma_{n}$. Substituting $q=r_{t} x, t=1, \ldots, n$ successively in (3.3); adding the resulting $n$ equations so obtained and then putting the expression $\sum_{t=1}^{n} G\left(r_{t} x\right)$ calculated from 3.3 . We obtain

$$
\begin{aligned}
\sum_{i=1}^{n} \sum_{t=1}^{n} G\left(p_{i} r_{t} x\right)-[G(x)-G(0)] \sum_{i=1}^{n} p_{i}^{\alpha} \sum_{t=1}^{n} r_{t}^{\alpha}-n^{2} G(0) \\
=E\left(p_{1}, \ldots, p_{n} ; x\right)+x^{\beta}\left\{\sum_{t=1}^{n} r_{t}^{\beta}\left[\sum_{i=1}^{n} G\left(p_{i}\right)+(m-n) G(0)\right]\right. \\
\left.+\sum_{i=1}^{n} p_{i}^{\alpha}\left[\sum_{t=1}^{n} G\left(r_{t}\right)+(m-n) G(0)\right]\right\}+E\left(r_{1}, \ldots, r_{n} ; x\right) \sum_{i=1}^{n} p_{i}^{\alpha}
\end{aligned}
$$

for all $x \in I,\left(p_{1}, \ldots, p_{n}\right) \in \Gamma_{n},\left(r_{1}, \ldots, r_{n}\right) \in \Gamma_{n}$. The left hand side of the above equation is commutative in $p_{i}$ and $r_{t}, i=1, \ldots, n ; t=1, \ldots, n$ (see p. 59, Acźel [1]). This implies the right hand side must also be commutative in $p_{i}$ and $r_{t}, i=1, \ldots, n ; t=1, \ldots, n$. As a result, we obtain the equation

$$
\begin{aligned}
& E\left(p_{1}, \ldots, p_{n} ; x\right) {\left[1-\sum_{t=1}^{n} r_{t}^{\alpha}\right]-E\left(r_{1}, \ldots, r_{n} ; x\right)\left[1-\sum_{i=1}^{n} p_{i}^{\alpha}\right] } \\
&=x^{\beta}\left\{\left[\sum_{t=1}^{n} r_{t}^{\alpha}-\sum_{t=1}^{n} r_{t}^{\beta}\right]\left[\sum_{i=1}^{n} G\left(p_{i}\right)+(m-n) G(0)\right]\right. \\
&\left.-\left[\sum_{i=1}^{n} p_{i}^{\alpha}-\sum_{i=1}^{n} p_{i}^{\beta}\right]\left[\sum_{t=1}^{n} G\left(r_{t}\right)+(m-n) G(0)\right]\right\} .
\end{aligned}
$$

Here, we notice that equation 3.5 depends on the parameters $\alpha$ and $\beta$. Therefore, we divide our discussion into two cases.

Case 1: $\alpha=\beta$

In this case, equation 3.5 reduces to

$$
E\left(p_{1}, \ldots, p_{n} ; x\right)\left[1-\sum_{t=1}^{n} r_{t}^{\beta}\right]=E\left(r_{1}, \ldots, r_{n} ; x\right)\left[1-\sum_{i=1}^{n} p_{i}^{\beta}\right] .
$$

Now, we assert that

$$
1-\sum_{t=1}^{n} r_{t}^{\beta} \not \equiv 0
$$

for fixed positive real power $\beta \neq 1$ satisfying 1.7). To the contrary, suppose that $1-\sum_{t=1}^{n} r_{t}^{\beta}=0$ for all $\left(r_{1}, \ldots, r_{n}\right) \in \Gamma_{n}$. By Lemma 1, there exists an additive mapping $a: \mathbb{R} \rightarrow \mathbb{R}$ so that $r^{\beta}=a(r)$ with $a(1)=1$. By Lemma 2, this holds when $\beta=0$ or $\beta=1$. Consequently we arrive at a contradiction as $\beta$ is presumed to be a fixed positive real power with $\beta \neq 1$, and hence (3.7) follows. Thus there exists a probability distribution $\left(r_{1}^{*}, \ldots, r_{n}^{*}\right) \in \Gamma_{n}$ so that $1-\sum_{t=1}^{n} r_{t}^{* \beta} \neq 0$. Using this in 3.6, we obtain

$$
E\left(p_{1}, \ldots, p_{n} ; x\right)=E_{0}(x)\left[1-\sum_{i=1}^{n} p_{i}^{\beta}\right]
$$


where $E_{0}: \mathbb{R} \rightarrow \mathbb{R}$ defined as $E_{0}(x)=\left[1-\sum_{t=1}^{n} r_{t}^{* \beta}\right]^{-1} E\left(r_{1}^{*}, \ldots, r_{n}^{*} ; x\right)$ is an additive mapping. Also from 3.4 , we get

$$
E_{0}(1)=-m G(0)
$$

From (3.3), 3.8) and (3.9) with $\alpha=\beta$, we obtain

$$
\sum_{i=1}^{n} \bar{G}\left(p_{i} q\right)-\bar{G}(q) \sum_{i=1}^{n} p_{i}^{\beta}-q^{\beta} \sum_{i=1}^{n} \bar{G}\left(p_{i}\right)=0
$$

where $\bar{G}: I \rightarrow R$ is defined as

$$
\bar{G}(x)=G(x)-E_{0}(x)-G(0)
$$

for all $x \in I$. Clearly $\bar{G}(0)=0$. On applying Lemma 1 on the functional equation $(3.10)$ and using $\bar{G}(0)=0$, it follows that there exists a mapping $E^{*}: \mathbb{R} \times I \rightarrow \mathbb{R}$, additive in the first variable so that

$$
\bar{G}(p q)-p^{\beta} \bar{G}(q)-q^{\beta} \bar{G}(p)=E^{*}(p ; q)
$$

with $E^{*}(1 ; q)=0$. The left hand side of $(3.12$ is symmetric in $p$ and $q$ (see Acźel [1]), so its right hand side must also be symmetric in $p$ and $q$. This implies the mapping $E^{*}$ is additive in the second variable also. Moreover from 3.12 , it can easily be verified that

$$
\begin{aligned}
\bar{G}(p q r)-p^{\beta} q^{\beta} \bar{G}(r)-q^{\beta} r^{\beta} \bar{G}(p)-r^{\beta} p^{\beta} \bar{G}(q) & =E^{*}(r ; p q)+r^{\beta} E^{*}(p ; q) \\
& =E^{*}(r p ; q)+q^{\beta} E^{*}(r ; p)
\end{aligned}
$$

for all $p \in I, q \in I, r \in I$.

Now, we proceed to show that $E^{*}(p ; q) \equiv 0$ on $I \times I$. On the contrary, suppose $E^{*}(p ; q) \neq 0$ on $I \times I$. This implies there exists some $p^{*} \in I, q^{*} \in I$ such that $E^{*}\left(p^{*} ; q^{*}\right) \neq 0$. From 3.13 , it follows that

$$
r^{\beta}=\left[E^{*}\left(p^{*} ; q^{*}\right)\right]^{-1}\left[E^{*}\left(r p^{*} ; q^{*}\right)+q^{* \beta} E^{*}\left(r ; p^{*}\right)-E^{*}\left(r ; p^{*} q^{*}\right)\right] .
$$

The right hand side of the above equation is additive, while its left hand side being a power mapping is multiplicative. Consequently by Lemma 2 , this holds only if $\beta=0$ or $\beta=1$. As a result we arrive at a contradiction and so $E^{*}(p ; q)$ vanishes identically on $I \times I$. With the aid of this 3.12 reduces to

$$
\bar{G}(p q)-p^{\beta} \bar{G}(q)-q^{\beta} \bar{G}(p)=0
$$

for all $p \in I, q \in I$. The general solution of 3.14 is $\bar{G}(p)=p^{\beta} \ell(p)$ for all $p \in I ; \ell: I \rightarrow \mathbb{R}$ being a logarithmic mapping. Hence from 3.11, the solution (3.1) is attained.

Case 2: $\alpha \neq \beta$

In this case, let $\left(z_{1}, \ldots, z_{n}\right) \in \Gamma_{n}$ and substitute $q=z_{t}, t=1, \ldots, n$ consecutively in (3.3). Then summing the resulting $n$ equations so obtained and using (3.4), it follows that

$$
\begin{aligned}
& \sum_{i=1}^{n} \sum_{t=1}^{n} G\left(p_{i} z_{t}\right)+m G(0)-n^{2} G(0)=\sum_{i=1}^{n} p_{i}^{\alpha}\left[\sum_{t=1}^{n} G\left(z_{t}\right)+(m-n) G(0)\right] \\
&+\sum_{t=1}^{n} z_{t}^{\beta}\left[\sum_{i=1}^{n} G\left(p_{i}\right)+(m-n) G(0)\right]
\end{aligned}
$$


for all $\left(p_{1}, \ldots, p_{n}\right) \in \Gamma_{n},\left(z_{1}, \ldots, z_{n}\right) \in \Gamma_{n}$. The left hand side of the above equation is commutative in $p_{i}$ and $z_{t}, i=1, \ldots, n ; t=1, \ldots, n$. Thus the commutativity on the right hand side yields

$$
\begin{aligned}
{\left[\sum_{i=1}^{n} G\left(p_{i}\right)+(m-n)\right.} & G(0)]\left[\sum_{t=1}^{n} z_{t}^{\alpha}-\sum_{t=1}^{n} z_{t}^{\beta}\right] \\
& =\left[\sum_{t=1}^{n} G\left(z_{t}\right)+(m-n) G(0)\right]\left[\sum_{i=1}^{n} p_{i}^{\alpha}-\sum_{i=1}^{n} p_{i}^{\beta}\right]
\end{aligned}
$$

for all $\left(p_{1}, \ldots, p_{n}\right) \in \Gamma_{n},\left(z_{1}, \ldots, z_{n}\right) \in \Gamma_{n}$. Now, we show that for $\alpha \neq \beta$

$$
\sum_{t=1}^{n} z_{t}^{\alpha}-\sum_{t=1}^{n} z_{t}^{\beta} \not \equiv 0
$$

where $\left(z_{1}, \ldots, z_{n}\right) \in \Gamma_{n}$ and $\alpha \neq 1, \beta \neq 1$ are fixed positive real powers satisfying (1.7). Suppose $\sum_{t=1}^{n} z_{t}^{\alpha}-$ $\sum_{t=1}^{n} z_{t}^{\beta}=0$ for all $\left(z_{1}, \ldots, z_{n}\right) \in \Gamma_{n}$. By Lemma 1 , there exists an additive mapping $a^{*}: \mathbb{R} \rightarrow \mathbb{R}$ so that $z^{\alpha}-z^{\beta}=a^{*}(z)$ with $a^{*}(1)=0$. By Lemma 3 , this holds when $\alpha=0, \beta=0$ or $\alpha=1, \beta=1$. Consequently we get a contradiction as $\alpha$ and $\beta$ are presumed to be fixed positive real powers with $\alpha \neq 1$ and $\beta \neq 1$. Thus (3.16) holds for $\alpha \neq \beta$ and so there exists a probability distribution $\left(z_{1}^{*}, \ldots, z_{n}^{*}\right) \in \Gamma_{n}$ such that $\sum_{t=1}^{n} z_{t}^{* \alpha}-\sum_{t=1}^{n} z_{t}^{* \beta} \neq 0$. Now choosing $0 \neq \sum_{t=1}^{n} z_{t}^{* \alpha}-\sum_{t=1}^{n} z_{t}^{* \beta}=d_{0}^{-1}$ (say) in the equation (3.15), we get

$$
\sum_{i=1}^{n} G\left(p_{i}\right)-d\left[\sum_{i=1}^{n} p_{i}^{\alpha}-\sum_{i=1}^{n} p_{i}^{\beta}\right]=(n-m) G(0)
$$

where $d=d_{0}\left[\sum_{t=1}^{n} G\left(z_{t}^{*}\right)+(m-n) G(0)\right] \in \mathbb{R}$ and $\left(p_{1}, \ldots, p_{n}\right) \in \Gamma_{n}$. By Lemma 1 , there exits an additive mapping $E_{1}: R \rightarrow R$ such that the solution 3.2 follows.

Proof of Theorem 3.1. Let us put $p_{1}=1, p_{2}=\cdots=p_{n}=0$ in the functional equation (A). We obtain

$$
\sum_{j=1}^{m}\left\{f\left(q_{j}\right)-g\left(q_{j}\right)-[h(1)+(n-1) h(0)] q_{j}^{\beta}\right\}=m(1-n) f(0)
$$

for all $\left(q_{1}, \ldots, q_{m}\right) \in \Gamma_{m}$. By Lemma 1, there exists an additive mapping $a: \mathbb{R} \rightarrow \mathbb{R}$ such that

$$
f(q)=g(q)+[h(1)+(n-1) h(0)] q^{\beta}+a(q)+f(0)-g(0)
$$

with

$$
a(1)=m[g(0)-n f(0)]
$$

From $\mathrm{A}, 3.17$ and 3.18 , we get

$$
\begin{aligned}
\sum_{i=1}^{n} \sum_{j=1}^{m} g\left(p_{i} q_{j}\right) & +[h(1)+(n-1) h(0)] \sum_{i=1}^{n} p_{i}^{\beta} \sum_{j=1}^{m} q_{j}^{\beta} \\
- & \sum_{i=1}^{n} p_{i}^{\alpha} \sum_{j=1}^{m} g\left(q_{j}\right)-\sum_{j=1}^{m} q_{j}^{\beta} \sum_{i=1}^{n} h\left(p_{i}\right)=m(n-1) g(0)
\end{aligned}
$$


for all $\left(p_{1}, \ldots, p_{n}\right) \in \Gamma_{n},\left(q_{1}, \ldots, q_{m}\right) \in \Gamma_{m} ; n \geq 3, m \geq 3$ being fixed integers. Now, substituting $q_{1}=$ $1, q_{2}=\cdots=q_{m}=0$ in 3.19 . We have

$$
\begin{aligned}
\sum_{i=1}^{n}\left\{g\left(p_{i}\right)+\right. & {\left.[h(1)+(n-1) h(0)] p_{i}^{\beta}-[g(1)+(m-1) g(0)] p_{i}^{\alpha}-h\left(p_{i}\right)\right\} } \\
= & (n-m) g(0)
\end{aligned}
$$

for all $\left(p_{1}, \ldots, p_{n}\right) \in \Gamma_{n}$. By Lemma 1, there exists an additive mapping $e: \mathbb{R} \rightarrow \mathbb{R}$ such that

$$
\begin{aligned}
h(p)= & g(p)+[h(1)+(n-1) h(0)] p^{\beta}-[g(1)+(m-1) g(0)] p^{\alpha} \\
& -e(p)-g(0)+h(0)
\end{aligned}
$$

with

$$
e(1)=n h(0)-m g(0) \text {. }
$$

Functional equation $(3.19)$ with $(3.20)$ and $(3.21)$ yields the functional equation $(\mathrm{B})$ where the mapping $G: I \rightarrow \mathbb{R}$ is defined as

$$
G(x)=g(x)-[g(1)+(m-1) g(0)] x^{\beta}
$$

for all $x \in I$. By Lemma 4 , mapping $G$ is of the form (3.1) and $(3.2)$. Thus solutions $\left(\alpha_{1}\right)$ (ii) and $\left(\alpha_{2}\right)($ ii) are immediate consequences of 3.1 , (3.2) and 3.22 with $G(0)=g(0)$.

From (3.20), (3.21), $\left(\alpha_{1}\right)$ (ii) and using $\alpha=\beta$; solution $\left(\alpha_{1}\right)$ (iii) follows by defining an additive mapping $E_{0}^{*}: \mathbb{R} \rightarrow \mathbb{R}$ as $E_{0}^{*}(x)=E_{0}(x)-e(x)$ with $E_{0}^{*}(1)=-n h(0)$. Further from (3.17), (3.18) and $\left(\alpha_{1}\right)(\mathrm{ii}) ;$

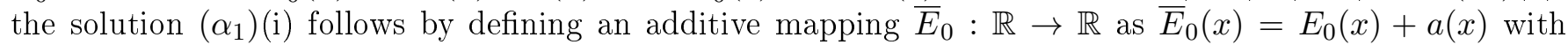
$\bar{E}_{0}(1)=-n m f(0)$. Similarly with the aid of $(3.20),(3.21)$ and $\left(\alpha_{2}\right)(i i)$; the solution $\left(\alpha_{2}\right)($ iii) is attained by defining an additive mapping $E_{1}^{*}: \mathbb{R} \rightarrow \mathbb{R}$ as $E_{1}^{*}(x)=E_{1}(x)-e(x)$ with $E_{1}^{*}(1)=-n h(0)$. Lastly, from (3.17), 3.18) and $\left(\alpha_{2}\right)$ (ii); the solution $\left(\alpha_{2}\right)$ (i) stands proved by defining an additive mapping $\bar{E}_{1}: \mathbb{R} \rightarrow \mathbb{R}$ as $\bar{E}_{1}(x)=E_{1}(x)+a(x)$ with $\bar{E}_{1}(1)=-n m f(0)$.

\section{Comments}

In this section our objective is to discuss the significance of the general solutions of functional equations (A) and $(\mathrm{B})$ in reference of information theory and diversity index.

In information theory the notion of entropy, which is referred as uncertainty was introduced by Shannon [27. The Shannon entropies $H_{n}: \Gamma_{n} \rightarrow \mathbb{R}, n=1,2, \ldots$ also known as additive entropies are defined as

$$
H_{n}\left(p_{1}, \ldots, p_{n}\right)=-\sum_{i=1}^{n} p_{i} \log _{2} p_{i}
$$

for all $\left(p_{1}, \ldots, p_{n}\right) \in \Gamma_{n}$ and $0 \log _{2} 0:=0$. A generalization of Shannon entropies 4.1 are the entropies $H_{n}^{\beta}: \Gamma_{n} \rightarrow \mathbb{R}, n=1,2, \ldots$ of degree $\beta(0<\beta \in \mathbb{R}, \beta \neq 1)$, which are defined as

$$
H_{n}^{\beta}\left(p_{1}, \ldots, p_{n}\right)=\left(1-2^{1-\beta}\right)^{-1}\left[1-\sum_{i=1}^{n} p_{i}^{\beta}\right]
$$

for all $\left(p_{1}, \ldots, p_{n}\right) \in \Gamma_{n}$ with $0^{\beta}:=0$ and $1^{\beta}:=1$. The entropies 4.2 are also referred as nonadditive entropies of degree $\beta(0<\beta \in \mathbb{R}, \beta \neq 1)$ in the literature and were introduced by Havrda and Charvát [5].

Index of diversity also known as diversity index is a real valued mapping defined on a probability distribution that highlights upon the differences in its sample space. Indeed, it is a nonnegative quantitative 
measure which is evolving with an integrative approach. Interestingly, there is plethora of definitions on diversity indices and several research papers reflect upon its applications. However, we would suggest the references [6], [7] and [26] to help the readers get an overview of this phenomenon and its areas of research.

For a probability distribution $\left(p_{1}, \ldots, p_{n}\right) \in \Gamma_{n}$, Hill [6] defined diversity number as

$$
N_{a}\left(p_{1}, \ldots, p_{n}\right)=\left(\sum_{i=1}^{n} p_{i}^{a}\right)^{1 / 1-a}
$$

where ' $n$ ' is referred as the richness; ' $a$ ' as the order of diversity and the term within the parentheses on the right hand side is called the basic sum. For $a=1$, the expression (4.3) is undefined. However, its limit as ' $a$ ' approaches to 1 exists and is related to Shannon entropy given by (4.1) (see p. 431, Hill [6]). Infact diversity indices corresponding to different values of ' $a$ ' have been discussed by Hill [6], Jost [7] and Tuomisto [30]. Moreover, Tuomisto has discussed the diversity index for $a>0, a<0$ and then mentioned that logically ' $a$ ' must be restricted to nonnegative values (see p. 5, [30]).

The general expression (4.3) is also called effective number or Hill number [7]. Clearly, the Hill number (4.3) satisfies the following postulates:

(i) $N_{a}\left(p_{1}, \ldots, p_{n}\right) \geq 0$.

(ii) $N_{a}$ attains maximum diversity for uniform distribution, i.e. when $p_{i}$ are equal.

(iii) $N_{a}$ attains minimum diversity for degenerate distribution, i.e. when $p_{i}=1$ and $p_{j}=0$ for $i \neq j$.

We notice that from 4.2 and 4.3 , it follows that

$$
\left[N_{a}\left(p_{1}, \ldots, p_{n}\right)\right]^{1-a}=1-\left(1-2^{1-a}\right) H_{n}^{a}\left(p_{1}, \ldots, p_{n}\right) .
$$

With the aid of (4.4), we present that $(1-a)^{t h}$ power of Hill number of order ' $a$ ' is related to the entropy of degree ' $a$ ' corresponding to the same value of ' $a$ '. Furthermore, by using (4.4) along with 1.8 we would be able to connect functional equations $(A)$ and $(B)$ with information theory and diversity index which is demonstrated as follows:

We begin by discussing the relevance of solutions of functional equation A. In view of the form of entropies given by (1.8), it is desirable to choose the logarithmic mapping $\ell: I \rightarrow \mathbb{R}$ as

$$
\ell(p)= \begin{cases}\delta \log _{2} p & \text { for } p \in] 0,1] \\ 0 & \text { for } p=0\end{cases}
$$

where $\delta$ is an arbitrary real constant.

Now, on choosing the logarithmic mapping $\ell: I \rightarrow \mathbb{R}$ as mentioned by (4.5), the solution $\left(\alpha_{1}\right)$ of A gives

$$
\begin{aligned}
\sum_{i=1}^{n} f\left(p_{i}\right)= & -\delta 2^{1-\beta} H_{n}^{(\beta, \beta)}\left(p_{1}, \ldots, p_{n}\right)+[g(1)+(m-1) g(0)+h(1) \\
& +(n-1) h(0)]\left[N_{\beta}\left(p_{1}, \ldots, p_{n}\right)\right]^{1-\beta}-n(m-1) f(0) \\
\sum_{i=1}^{n} g\left(p_{i}\right)= & -\delta 2^{1-\beta} H_{n}^{(\beta, \beta)}\left(p_{1}, \ldots, p_{n}\right)+[g(1)+(m-1) g(0)] \\
& \times\left[N_{\beta}\left(p_{1}, \ldots, p_{n}\right)\right]^{1-\beta}+(n-m) g(0)
\end{aligned}
$$

and

$$
\begin{aligned}
\sum_{i=1}^{n} h\left(p_{i}\right)= & -\delta 2^{1-\beta} H_{n}^{(\beta, \beta)}\left(p_{1}, \ldots, p_{n}\right) \\
& +[h(1)+(n-1) h(0)]\left[N_{\beta}\left(p_{1}, \ldots, p_{n}\right)\right]^{1-\beta} .
\end{aligned}
$$


Hence it can be concluded that mappings $f, g$ and $h$ of solution $\left(\alpha_{1}\right)$ are connected to entropies of type $(\alpha, \beta)$ for $\alpha=\beta$ and Hill number of order $\beta$ if $\delta \neq 0$. Moreover if $\delta=0$, then all three mappings $f, g$ and $h$ are connected to Hill number of order $\beta$.

Next we compute the summands of the solution $\left(\alpha_{2}\right)$ of $\mathrm{A}$.

$$
\begin{aligned}
\sum_{i=1}^{n} f\left(p_{i}\right)= & d\left(2^{1-\alpha}-2^{1-\beta}\right) H_{n}^{(\alpha, \beta)}\left(p_{1}, \ldots, p_{n}\right)+[g(1)+(m-1) g(0) \\
& +h(1)+(n-1) h(0)]\left[N_{\beta}\left(p_{1}, \ldots, p_{n}\right)\right]^{1-\beta} \\
& -n(m-1) f(0) \\
\sum_{i=1}^{n} g\left(p_{i}\right)= & d\left(2^{1-\alpha}-2^{1-\beta}\right) H_{n}^{(\alpha, \beta)}\left(p_{1}, \ldots, p_{n}\right)+[g(1)+(m-1) g(0)] \\
& \times\left[N_{\beta}\left(p_{1}, \ldots, p_{n}\right)\right]^{1-\beta}+(n-m) g(0)
\end{aligned}
$$

and

$$
\begin{aligned}
\sum_{i=1}^{n} h\left(p_{i}\right)= & \{d-[g(1)+(m-1) g(0)]\}\left(2^{1-\alpha}-2^{1-\beta}\right) H_{n}^{(\alpha, \beta)}\left(p_{1}, \ldots, p_{n}\right) \\
& +[h(1)+(n-1) h(0)]\left[N_{\beta}\left(p_{1}, \ldots, p_{n}\right)\right]^{1-\beta} .
\end{aligned}
$$

It follows that if $d \neq 0$, then mappings $f, g$ and $h$ of solution $\left(\alpha_{2}\right)$ are connected to entropies of type $(\alpha, \beta)$ for $\alpha \neq \beta$ and Hill number of order $\beta$. Also if $d=0$, then mappings $f$ and $g$ are connected to Hill number of order $\beta$ only, while mapping $h$ is connected to entropies of type $(\alpha, \beta)$ for $\alpha \neq \beta$ and Hill number of order $\beta$.

Thus, functional equation $\mathrm{A}$ is related to information theory and diversity index.

Similarly, we discuss the relevance of solutions of functional equation $(\mathrm{B})$ with information theory and diversity index. On computing the summands $(3.1)$ and $(3.2)$ of $(\bar{B})$ and using $(4.5)$, it follows that

$$
\sum_{i=1}^{n} G\left(p_{i}\right)=-\delta 2^{1-\beta} H_{n}^{(\beta, \beta)}\left(p_{1}, \ldots, p_{n}\right)+(n-m) G(0)
$$

and

$$
\sum_{i=1}^{n} G\left(p_{i}\right)=d\left(2^{1-\alpha}-2^{1-\beta}\right) H_{n}^{(\alpha, \beta)}\left(p_{1}, \ldots, p_{n}\right)+(n-m) G(0) .
$$

It can be concluded that if $\delta \neq 0$ and $d \neq 0$, then solutions of functional equation $(\mathrm{B}$ are connected to entropies of type $(\alpha, \beta)$ for $\alpha=\beta$ and entropies of type $(\alpha, \beta)$ for $\alpha \neq \beta$ respectively. The cases $\delta=0$ and $d=0$ are not of much relevance as $\sum_{i=1}^{n} G\left(p_{i}\right)$ do not represent any form of entropies. Thus the functional equation $\sqrt{\mathrm{B}}$ ) is also emerging from information theory.

This paper is not only of interest to the information theorist who are involved in discovering new measures of information or entropy but also to the researchers who are working in the field of functional equations and focussed on exploring new equations and their applications. In future, building upon the work of Light and Margolin [12], Margolin and Light [15] we can reflect upon the applications of sum form functional equations for data analysis in various fields. This paper will be indispensable to those researchers who opt for an interdisciplinary research methodology that aims to not only add new dimensions to their field of research but also includes efforts to establish its connect with Functional Equations, Information Theory and Statistics.

Acknowledgement. The authors are grateful to the referee(s) for his/her valuable suggestions. 


\section{References}

[1] J. Aczél, Lectures on Functional Equations and Their Applications, Academic Press New York and London, 1966.

[2] M. Behara and P. Nath, Information and entropy of countable measurable partitions I, Kybernetika, 10 (1974), $491-503$.

[3] Z. Daróczy and L. Losonczi, Über die Erweiterung der auf einer Punktmenge additiven Funktionen, Publicationes Mathematicae (Debrecen), 14 (1967), 239-245.

[4] C. Gini, Variabilitá e Mutabilitá, Studi Economicoaguridici della facotta di Giurisprudenza dell, Universite di Cagliari III, Parte II, 1912.

[5] J. Havrda and F. Charvát, Quantification method of classification processes. Concept of structural $\alpha$-entropy, Kybernetika, 3 (1967), 30-35.

[6] M.O. Hill, Diversity and evenness: A unifying notation and its consequences, Ecology, 54(2) (1973), 427-432.

[7] L. Jost, Entropy and diversity, Oikos, 113(2) (2006), 363-375.

[8] Pl. Kannappan, An application of a differential equation in information theory, Glasnik Matematicki, 14(34) (1979), 269274.

[9] Pl. Kannappan, On a generalization of sum form functional equation III, Demonstratio Mathematica, 13 (1980), $749-754$.

[10] I. Kocsis, On the stability of a sum form functional equation of multiplicative type, Acta Acad. Paed. Agriensis, Sectio Mathematicae, 28 (2001), 43-53.

[11] R.C. Lewontin, The apportionment of human diversity, Evolutionary Biology, 6 (1972), 381-398.

[12] R.J. Light and B.H. Margolin, An analysis of variance for categorical data, J. Amer. Stat. Asso., 66 (1971), $534-544$.

[13] L. Losonczi and Gy. Maksa, The general solution of a functional equation of information theory, Glasnik Matematicki, 16(36) (1981), 261-268.

[14] L. Losonczi and Gy. Maksa, On some functional equations of the information theory, Acta Mathematica Academiae Scientiarum Hungarica, 39(1-3) (1982), 73-82.

[15] B.H. Margolin and R.J. Light, An analysis of variance for categorical data, II: small sample comparisons with chi square and other competitors, J. Amer. Stat. Asso., 69 (1974), 755-764.

[16] A.M. Mood, F.A. Graybill and D.C. Boes, Introduction to the Theory of Statistics, 3rd Edition, McGraw-Hill, New York, 1974.

[17] P. Nath and D.K. Singh, On a multiplicative type sum form functional equation and its role in information theory, Applications of Mathematics, 51(5) (2006), 495-516.

[18] P. Nath and D.K. Singh, A sum form functional equation related to various entropies in information theory, Glasnik Matematicki, 43(63) (2008), 159-178.

[19] P. Nath and D.K. Singh, On a sum form functional equation related to entropies and some moments of a discrete random variable, Demonstratio Mathematica, 42(1) (2009), 83-96.

[20] P. Nath and D.K. Singh, On a sum form functional equation related to entropies of type ( $\alpha, \beta)$, Asian-European Journal of Mathematics, 6(2) (2013), 13 pages.

[21] P. Nath and D.K. Singh, On a functional equation arising in statistics, Kragujevac Journal of Mathematics, 39(2) (2015), 141-148.

[22] P. Nath and D.K. Singh, Some functional equations and their corresponding sum forms, Sarajevo Journal of Mathematics, 12(24) (2016), 89-106.

[23] P. Nath and D.K. Singh, On a sum form functional equation containing five unknown mappings, Aequationes Mathematicae, 90 (2016), 1087-1101.

[24] P. Nath and D.K. Singh, On an equation related to nonadditive entropies in information theory, Mathematics for Applications, 6(1) (2017), 31-41.

[25] E.C. Pielou, Ecological Diversity, Wiley, New York, 1975.

[26] C.R. Rao, Diversity and dissimilarity coefficients: A unified approach, Theoretical Population Biology, 21 (1982), $24-43$.

[27] C.E. Shannon, A mathematical theory of communication, Bell System Technical Journal, 27 (1948), 379-423; 623-656.

[28] D.K. Singh and P. Dass, On a functional equation related to some entropies in information theory, Journal of Discrete Mathematical Sciences and Cryptography, 21(3) (2018), 713-726.

[29] D.K. Singh and S. Grover, On the stability of a sum form functional equation related to nonadditive entropies, Journal of Mathematics and Computer Science, 23(4) (2021), 328-340.

[30] H. Tuomisto, A diversity of beta diversities: straightening up a concept gone awry. Part 1 . Defining beta diversity as a function of alpha and gamma diversity, Ecography, 33(1) (2010), 2-22. 